\title{
NEGATIVE MARKETING EFFECT ON UTILIZATION OF UNSAFE PRODUCTS
}

\begin{abstract}
Abdul Aziz Afzal Siddiqui ${ }^{1}$
ABSTRACT

Firms that intend to be ethically responsible for producing and promoting products that are accountable as unsafe products. These products are not physically, socially, and ethically safe as they create significant risk or injury to the person. It not only harms the person but also the others in the surrounding and cause damage to society. It includes cigarettes, cigars, and pipe tobacco are made from dried tobacco leaves which have a health impact on a bystander. Similarly, Firm attempt to put efforts aimed at discouraging (not destroying) the demand for such products and indulge in negative marketing. Common negative marketing strategies include higher prices, scaled-down advertising, and product redesign. This paper investigates the impact of negative marketing practices on unsafe products.
\end{abstract}

Keywords: Unsafe Products; Negative Marketing; Social Norms; Health impact on Bystanders.

\section{INTRODUCTION}

In this research, we will bring in to light a few elements which are determining and leading to certain outcome regarding the negative marketing of unsafe products. There are many unsafe products such as wine, narcotics, weapons, tobacco etc. As we live in an eastern country, so our culture does not support much more wine, narcotics, and weapon whereas tobacco is normally used in our country although it is also injurious to health due to low price or easily accessible product, we are going to research on tobacco only from all unsafe products.

Negative marketing is an effort or tool to reduce or bound demand for consumption of a particular product or service on a permanent or temporary basis. Therefore, de-marketing is

\footnotetext{
${ }^{1}$ Engro Energy Limited. Email: Afzaal_siddiqui@hotmil.com
} 
able to be applied on both private and public sector goals. Injurious goods are sometime being promoted to the consumers by manufacturer and their distributors (Raut \& Pawar, 2012).

Demarketing strategies have played a significant, if unacknowledged, role in the global tobacco war. The authors used structural equation modeling to test the hypothesized relationships among policy initiatives aimed at each of the 4Ps and attitudes toward smoking, attitudes toward the tobacco industry and intentions to quit smoking at two points in time. (Lefebvre \& Kotler, 2011). The research presented adds to the growing body of literature on demarketing efforts by examining the linkages between their implementation and consumers' subsequent attitudinal and behavioral responses (Ramirez et al., 2017).

\section{STATEMENT OF THE PROBLEM}

In this research, we would study and discuss factors that lead to utilization of unsafe products i.e., tobacco. Problem statement will help to discover factors of utilization of tobacco. Dependent variable is utilization of unsafe products i.e., tobacco whereas it will be found, in this research, which factors are playing role of independent variables on utilization of tobacco.

\section{RESEARCH QUESTIONS}

Research question are questions those a research project sets out to answer. Choosing research questions is an essential element of both quantitative and qualitative research. Investigation will require data collection and analysis, and the methodology for this will vary widely. Our research questions are as follows.

a) Does negative marketing effect on end user psychologically? Or they think it is useless. What are their attitudes towards advertisement of tobacco products?

b) Is there any connection between social gathering and utilization of tobacco?

c) Is advertisement of tobacco in films and give away by tobacco companies discourage negative marketing of tobacco?

d) Does negative marketing of tobacco will help to reduce utilization of tobacco?

e) At which extent negative marketing demoralizes intention towards utilization of tobacco?

\section{RESEARCH OBJECTIVES}

The objective of this hypothesis testing is to highlight the concept of association of utilization of tobacco on negative marketing tactics. Objective of this hypothesis testing is to check any 
correlational theory between negative marketing and utilization of unsafe products i.e., tobacco. If we increase negative marketing so utilization of tobacco is decreased or not.

\section{SIGNIFICANCE OF THE STUDY}

Negative marketing or demarketing may be considered as non-selling or marketing in reverse, which includes general and selective demarketing. Although the concept of demarketing or negative marketing lacks a precise theoretical definition, it refers to an attempt by the firm to discourage all or some of its customers from making purchases either temporarily or permanently. Since the initial interests in the subject area of how to market strategically in times of shortages began, different viewpoints have been offered as to how the firm should pursue demarketing. Significance of the study with concern of tobacco product negative marketing. Another objective of the study is to discover the perception of tobacco users towards the negative marketing of tobacco products.

Various articles indicate that the study is useful to hypothesis testing in their countries and respective territories such as USA, UK, UAE and in India as well. Marketing scholars have studied demarketing primarily in the context of smoking. This research enlarges set of hypotheses desired at understanding the impact of negative marketing of unsafe product i.e., Tobacco. (Raut \& Pawar, 2012)

There are many unsafe products such as wine, narcotics, weapons, tobacco etc. As we live in eastern country, so our culture does not support much more wine, narcotics and weapon whereas tobacco is normally used in our country although it is also injurious to health but due to low price or easy accessible product, we are going to research on the utilization of tobacco products only from all unsafe products.

\section{LIMITATIONS OF STUDY}

Everything has limitations in their scope thus this study also has few limitations which are described as follows.

a) Although we are not gathering data from any person but just to reach any particular and certain conclusion, so we assume that as we live in a very populated country even the city is overpopulated so we would gather data from the city of Karachi and few areas of Karachi.

b) There are many people who use tobacco products so it would be complicated to get data from majority for the sake of minimizing sample error. 
c) Moreover, this study does not have destructive nature of sample, but it has more timeconsuming activity and large population size that is why we account for these elements as limitations in our study.

\section{SCOPE OF THE STUDY}

Although we are not gathering data from any person but just to reach any particular and certain conclusion, we assume that we are going to collect data from KHI and some of its areas. This study is not specific to any gender thus male and female both are tested and checked for hypothesis testing. As such no special educational background is necessary. The study will cover both literate and illiterate people, but we have age restriction in this research. We assume that we will collect data from person who is must above 25 and below 35 and we also assume that we are supposed to do hypothesis testing on 384 peoples regardless of their class of income. It includes lower class, middle class and upper class.

\section{RESEARCH ASSUMPTIONS}

Even though the data is not gathered from any person but just to reach a particular and certain conclusion, we assumed that we would gather data from people who utilize tobacco products. Only who are addictive or habitual to do smoking we will gather data from them as well as we will ask them the reason for smoking. What makes them to smoke more, and in which circumstances they do more, and any negative marketing is going to impact on utilization of tobacco products what they perceive about their selves and regarding other people. 
Definition of Key Words

\section{LITERATURE REVIEW}

Demarketing of tobacco has primarily occurred along three fronts mass Media i.e., the use of antismoking advertisements, regulatory measures i.e., taxes and smoking bans, and public opinion i.e., normative behavior. The goal of mass media efforts of governments and some tobacco manufacturers has been to discourage and prevent smoking behavior. The goal of many of these social marketing campaigns has been to prevent adolescents from starting to smoke cigarettes. Findings concerning antismoking advertising have shown that exposure of students to these ads has contributed to the enhancement of school-based prevention programs (Moore, 2005).

Media and advertising play a significant role in marketing tobacco products to the public. Tobacco companies use strategic marketing campaigns and enticing advertisements to encourage customers to purchase specific brands of tobacco products. In order to create a foundation for an effective stop smoking intervention, the attitudes and beliefs of the indigent must be considered. Unfortunately, there have been very few studies conducted on the attitudes and beliefs regarding tobacco use among the poor (Lee et al., 2004).

A ban on cigarette advertising through television and radio has been in place in the U.S. since 1971. However, Saffer and Chaloupka (2000) conclude that governmental demarketing of tobacco through advertising bans in the U.S. will have little effect on tobacco consumption unless a comprehensive ban is enforced. This research advances demarketing research in an anti-consumption context by empirically assessing the influence of demarketing measures on smokers' attitudes and their intention to quit smoking. Specifically, two waves of a survey were used to test the effect of smokers' elaboration as a consequence of the four demarketing elements, product, price, place, and promotion. To fulfill this purpose this article (1) develops a conceptual model in which the 4Ps of demarketing are treated as determinants of smokers' attitude and quitting intention, (2) formulates testable hypotheses on direct, mediating and longitudinal effects, and (3) tests the model against empirical data from two survey waves, based on the same sample of respondents. (Shiu et al., 2009)

Prevention, Protection and Prosecution can also be considered as tools of de-marketing. The de-marketing campaign has resulted in increased negative attitudes toward smoking and a stigma toward individuals who smoke (Kim \& Shanahan; Pechmann \& Ratneshwar, 1994). 
Antismoking advertising alone is not responsible for changes in perceptions of smoking. Researchers (e.g., McAlister, Krosnick, \& Milburn, 1984; Meier, 1991) have long noted that negative public sentiment as expressed through parents, peers, and siblings are important factors in the formation of unfavorable attitudes toward smoking. (Raut \& Pawar, 2012)

Demarketing, counter-marketing, and social marketing may all sound similar though they are different constructs. So, it may be worthwhile to briefly distinguish them from one another before going on with demarketing. As alluded to above, demarketing efforts involve an organization's public advocacy for consumption reduction (Kotler 2011). As Gundlach, Bradford, and Wilkie (2010, p. 107) note, "demarketing involves discouraging demand in general or on the part of a certain class of customers, either temporarily or on a continuing basis. Counter-marketing, a stronger strategy, involves total repudiation of the relevant demand, in getting rid of undesirable customers certain types of transactions. (Ramirez et al., 2017)

Demarketing strategies have played a significant, if unacknowledged, role in the global tobacco war. While many participants would not be familiar with the term, demarketing objectives and strategies are readily apparent. In the tobacco control field, 'counter-marketing' has been used to describe promotional efforts such as mass media campaigns (Farrelly et al., 2002). However, other tobacco control efforts aimed at product, price and place restrictions have rarely been conceptualized into a marketing mix framework, with the exception of the ASSIST project as described by Lefebvre (2008). (Lefebvre \& Kotler, 2011)

Demarketing strategy is an integral part of overall marketing and focuses on reducing or rationing demand. The market situation that has accelerated interest in demarketing offers new opportunities to the alert marketer and important opportunities for all companies to increase the productivity of their marketing expenditures. ( Dadzie, 1989; Cui, 1974) 


\section{THEORETICAL FRAMEWORK}

\begin{tabular}{|c|c|c|}
\hline $\begin{array}{c}\text { Demarketing/ } \\
\text { Negative Marketing }\end{array}$ & 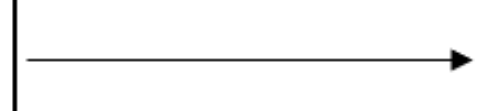 & $\begin{array}{c}\text { Utilization of } \\
\text { Tobacco }\end{array}$ \\
\hline
\end{tabular}

\section{RESEARCH HYPOTHESES}

H1: There is negative/inverse relationship between utilization of tobacco and Negative Marketing.

H2: There is no relationship between Utilization of tobacco and Negative Marketing.

\section{RESEARCH METHODOLOGY}

\section{Research Design}

This research is quantitative in nature as various theories related to the subject already exist and available. This research just attempt to test the hypotheses and relationship between dependent variable i.e., utilization of tobacco and independent variable i.e., negative marketing.

This study is longitudinal in nature. As a longitudinal study is a research design that involves repeated observations of the same variables over short or long periods of time, so our research time horizon is longitudinal. Moreover, the study setting of this research is non-contrived or natural. All variables are kept as per practice, standard, or as per routine as they were. This research is done in natural environment where work proceed normally.

Similarly, as a philosophy of research, this research pertains to positivism because it is quantitative research. Positivism adheres to the view that only factual knowledge gained through observation, including measurement, is trustworthy. In positivism studies the role of the researcher is limited to data collection and interpretation in an objective way.

\section{Population and Sample}

\section{Sample Unit}

Sample unit is an individual person. The term sampling unit refers to a singular value within a sample database. Although we are not gathering data from any person but just to reach any 
particular and certain conclusion, we assume that we will count a person as an individual sample unit.

\section{Sampling Technique}

Although we did not gather data from any person but just to reach any particular and certain conclusion, we assume that we don't have data or available list of people who utilizes tobacco so this type of sampling technique is called non-probability sampling technique as we don't have certain or known population size.

Sample Size

The data was collected from 384 people and the response rate was $70 \%$. The researcher used confidence level of $95 \%$ and confidence interval is 5 .

\section{Instrumentation}

\section{Variables}

In this research we have one dependent variable i.e., Utilization of Tobacco. It is observed a variable. It will be measured by sales of tobacco before and after negative marketing so that we could check effect of negative marketing in term of increase in sales or decrease in sales unit of tobacco and we have one independent variable i.e., negative marketing/demarketing. It is un-observed variable. It will be measured by questionnaire that we have designed that negative marketing does effect on utilization of tobacco or not. Items are given in appendix. A descriptive research design, involving 384 samples size was used for this study. A structured questionnaire was used as research instrument. It was pre-tested for further refinement. The Likert scale type statements were generated to measure the perception of tobacco users towards the negative marketing of tobacco products on a simple five-point scale, preferred for ease of administration in short time. The points on the scale were labeled as, 1 (strongly disagree), to 5 (strongly agree) (Raut \& Pawar, 2012).

\section{Validity Test}

Validity test is the extent to which a test accurately measures what it is supposed to measure. Validity test refers to the degree to which evidence and theory support the interpretations of test scores entailed by proposed uses of tests. 


\section{Reliability Test}

Reliability test refers to the degree to which a test is consistent and stable in measuring what it is intended to measure. Most simply put, a test is reliable if it is consistent within itself and across time.

\section{ETHICAL CONSIDERATIONS}

Ethical considerations were maintained while conducting this research. We assured that in all processes of this research, respect for the dignity of research participants should be prioritized. Any deception or exaggeration about the aims and objectives of the research must be avoided. Any type of misleading information, as well as representation of primary data findings in a biased way must be avoided. We also assured that full consent should be obtained from the participants prior to the study. Anonymity of individuals who are participating in the research, has to be ensured. Any type of communication in relation to the research should be done with honesty and transparency. The protection of the privacy of research participants has to be ensured. Affiliations in any forms, sources of funding, as well as any possible conflicts of interests have to be declared and we also assume that we will ensure adequate level of confidentiality of the research data.

\section{DATA ANALYSES}

\section{Data Analysis Plan}

A data analysis plan is a roadmap for how we organize and analyze survey data and it should help to achieve objectives that relate to the goal we set before we started survey. Data collection method is primary data collection as we are collecting data via questionnaire. We intended to use SPSS software for data analysis. We also plan to use different statistical tools to measure frequency, factor analysis, reliability analysis, descriptive statistics, hypothesis testing and many other advanced tools so based on the statistical result, we derived the proper interpretation and conclusion.

\section{RESULTS}

\section{Descriptive Statistics}

Descriptive statistic is a summary statistic that quantitatively describes and summarizes features from a collection of information. Although we did not gather data from any person but just to reach any particular and certain conclusion, we assume that we will collect data from person who is must above 25 and below 35 and 50 pc population is less than 30 years and 
remaining $50 \mathrm{pc}$ population is more than 30 years and we also assume that we are supposed to do hypothesis testing on 384 peoples. 80 pc of 384 people is male whereas remaining 20 pc population is female. 25 pc population cover the lower-class participants, 50 pc population covers the middle-class participants and remaining 25 pc population cover the upper-class participants. Whereas all, 100 pc population will be based on different areas of Karachi.

\section{Inferential Statistics}

Inferential statistics allows to make predictions or inferences from that data with inferential statistics, we take data from samples and generalize about a population. It is a conclusion reached on the basis of evidence and reasoning. Although we did not gather data from any person but just to reach any particular and certain conclusion, we assume that we have collected data from our sample. First, we would account for estimating parameters. This means taking a statistic from sample data i.e., sample mean and using it to say something about a population parameter i.e., population mean. Secondly, we would describe hypothesis tests. This is where we can use sample data to answer research questions, so we assume that we conclude this research based on sample data gather from participants we reached at the point that our research hypothesis 1 is accepted because sig value is $<0.05$ and we also assume that we used different statistical tools to measure frequency, factor analysis, reliability analysis, descriptive statistics, correlational value, hypothesis testing and many other advanced tools so based on the statistical result Hypothesis 1 is accepted. It is alternative hypothesis and Hypothesis 2 is rejected it is null hypothesis.

H1: There is negative/inverse relationship between utilization of tobacco and Negative Marketing. (Alternative Hypothesis).

H2: There is no relationship between Utilization of tobacco and Negative Marketing.

\section{HYPOTHESIS ASSESSMENT SUMMARY}

Our first research hypothesis 'There is negative/inverse relationship between utilization of tobacco and Negative Marketing' is accepted because we assume that we used different statistical tools to measure frequency, factor analysis, reliability analysis, descriptive statistics, hypothesis testing and many other advanced tools so based on the statistical result Hypothesis 1 is accepted whereas hypothesis 2 'There is no relationship between Utilization of tobacco and Negative Marketing' is rejected. 


\section{DISCUSSION, CONCLUSION AND RECOMMENDATIONS}

We conducted research on negative marketing and its effect on utilization of unsafe products. We choose tobacco from unsafe products. The tobacco industry uses a variety of different marketing and promotion tools to influence customer preference and increase the rate of new customers. All major businesses utilize marketing and promotional plans to promote their product to the public. The advertising goal of the company is to make the product appear desirable to a specific population in order to increase the sales of the product. In the case of tobacco companies, there are many different marketing and promotional strategies that are used to sell tobacco products to indigent populations.

After conducting complete research, we assume that we concluded this research based on sample data gather from participants we reached at the point that there is negative/inverse relationship between utilization of tobacco and Negative Marketing. The presentation of cigarettes in films is also important to the tobacco industry as it communicates continued social acceptability of smoking. The tobacco industry has had a long-term relationship with Hollywood. Study showed that $87 \%$ of the movies contained at least one incidence of tobacco use, $28 \%$ contained a minimum of one brand appearance, $8 \%$ had actor/actress endorsements, and $24 \%$ had background tobacco appearances. (Lee et al., 2004)

Furthermore, tobacco industry gains additional promotional advertising by giving away free items to the public with the tobacco company's brand or logo represented on the free item. Another study indicated that young adults between the ages of 25 and 32 were the most likely to own a tobacco promotional item, and the willingness to use a promotional item was highest in adolescents 20 to 22 years of age. Recommendation of this report is to ban on presentation of tobacco in films as it will useless that at one side, we are discouraging utilization on tobacco but at the same time we are continuing social acceptability of utilization of tobacco. Government should account for the investor who invest in the promotional activities by the tobacco industry. This part of promotional strategy should also be observed that few tobacco companies distribute give away such as t-shirts, hats, lighters, bags, diaries etc. to its customers and these are labelled by tobacco products and brand specified for the sake of advertisement. Obtaining a sports bag, an item of clothing, or any other kind of gear with a cigarette brand logo on it leads to reliably predicts the progression to established smoking in adolescents this form of advertising also escapes the mandatory health warning and may create the impression that tobacco products are socially acceptable, and it indicates that there is tolerance in the 
society for tobacco products. (Lee et al., 2004). These kinds of promotional activities must be avoided to demotivate utilization of tobacco. If these advertisements are not going to stop by Government, so there will be no significant change on utilization of tobacco by negative marketing of tobacco products. 


\section{REFERENCES}

Abratt, R., \& Sacks, D. (1988). The marketing challenge: towards being profitable and socially responsible. Journal of Business Ethics, 7(7), 497-507.

Cui, D. (1974). Demarketing. 37(October), 51-57.

Dadzie, K. Q. (1989). Demarketing strategy in shortage marketing environment. Journal of the Academy of Marketing Science, 17(2), 157-165.

Kotler, P., \& Gertner, D. (2002). Country as brand, product, and beyond: A place marketing and brand management perspective. Journal of brand management, 9(4), 249-261.

Lee, D., Cutler, B. D., \& Burns, J. (2004). The marketing and demarketing of tobacco products to low-income African-Americans. Health Marketing Quarterly, 22(2), 51-68. https://doi.org/10.1300/J026v22n02_04

Lefebvre, R. C., \& Kotler, P. (2011). Design thinking, demarketing and behavioral economics: Fostering interdisciplinary growth in social marketing. The SAGE Handbook of Social Marketing, January, 80-94. https://doi.org/10.4135/9781446201008.n6

Magno, F. (2012). Managing product recalls: The effects of time, responsible vs. opportunistic recall management and blame on consumers' attitudes. Procedia-Social and Behavioral Sciences, 58, 1309-1315.

Moore, R. S. (2005). The sociological impact of attitudes toward smoking: Secondary effects of the demarketing of smoking. Journal of Social Psychology, 145(6), 703-718. https://doi.org/10.3200/SOCP.145.6.704-718

Ramirez, E., Tajdini, S., \& David, M. E. (2017). The Effects of Proenvironmental Demarketing on Consumer Attitudes and Actual Consumption. Journal of Marketing Theory and Practice, 25(3), 291-304. https://doi.org/10.1080/10696679.2017.1311219 
Raut, U., \& Pawar, P. (2012). An analysis of perception of tobacco consumers towards the deMarketing of tobacco product(s). Abhinav National Monthly Refereed Journal Of Research In Commerce \& Management, 1, Vol.1, Issue .10, pp. 114-121,.

Schwartz, V. E. (1985). Unavoidably Unsafe Products: Clarifying the Meaning and Policy Behind Comment K. Wash. \& Lee L. Rev., 42, 1139.

Shiu, E., Hassan, L. M., \& Walsh, G. (2009). Demarketing tobacco through governmental policies - The 4Ps revisited. Journal of Business Research, 62(2), 269-278. https://doi.org/10.1016/j.jbusres.2008.01.034 